\title{
Differential Contribution of Plant-Beneficial Functions from Pseudomonas kilonensis F113 to Root System Architecture Alterations in Arabidopsis thaliana and Zea mays
}

\author{
Jordan Vacheron, ${ }^{1}$ Guilhem Desbrosses, ${ }^{2}$ Sébastien Renoud, ${ }^{1}$ Rosa Padilla, ${ }^{1}$ Vincent Walker, ${ }^{1}$ \\ Daniel Muller, ${ }^{1}$ and Claire Prigent-Combaret ${ }^{1,+}$ \\ ${ }^{1}$ UMR Ecologie Microbienne, CNRS, INRA, VetAgro Sup, UCBL, Université de Lyon, 43 bd du 11 Novembre, F-69622 \\ Villeurbanne, France; and ${ }^{2}$ CNRS, INRA, UMR5004, Biochimie \& Physiologie Moléculaire des Plantes, Montpellier, France
}

Accepted 15 September 2017.

\begin{abstract}
Fluorescent pseudomonads are playing key roles in plantbacteria symbiotic interactions due to the multiple plantbeneficial functions (PBFs) they are harboring. The relative contributions of PBFs to plant-stimulatory effects of the wellknown plant growth-promoting rhizobacteria Pseudomonas kilonensis F113 (formerly P. fluorescens F113) were investigated using a genetic approach. To this end, several deletion mutants were constructed, simple mutants $\Delta p h l D$ (impaired in the biosynthesis of 2,4-diacetylphloroglucinol [DAPG]), $\Delta$ acdS (deficient in 1-aminocyclopropane-1-carboxylate deaminase activity), $\Delta$ gcd (glucose dehydrogenase deficient, impaired in phosphate solubilization), and $\Delta$ nirS (nitrite reductase deficient), and a quadruple mutant (deficient in the four PBFs mentioned above). Every PBF activity was quantified in the wild-type strain and the five deletion mutants. This approach revealed few functional interactions between PBFs in vitro. In particular, biosynthesis of glucose dehydrogenase severely reduced the production of DAPG. Contrariwise, the DAPG production impacted positively, but to a lesser extent, phosphate solubilization. Inoculation of the F113 wild-type strain on Arabidopsis thaliana Col-0 and maize seedlings modified the root architecture of both plants. Mutant strain inoculations revealed that the relative contribution of each PBF differed according to the measured plant traits and that F113 plantstimulatory effects did not correspond to the sum of each PBF relative contribution. Indeed, two PBF genes ( $\Delta a c d S$ and $\Delta$ nirS) had a significant impact on root-system architecture from both model plants, in in vitro and in vivo conditions. The current work underscored that few F113 PBFs seem to interact between each other in the free-living bacterial cells, whereas they control in concert Arabidopsis thaliana and maize growth and development.
\end{abstract}

Current address for Jordan Vacheron: Université de Lausanne, Département de Microbiologie Fondamentale, Quartier UNIL-Sorge, CH-1015 Lausanne, Switzerland

${ }^{\dagger}$ Corresponding author: Claire Prigent-Combaret;

E-mail: claire.prigent-combaret@univ-lyon1.fr

*The $\boldsymbol{e}$-Xtra logo stands for "electronic extra" and indicates that four supplementary figures and five supplementary tables are published online.

@ 2018 The American Phytopathological Society
Plant growth-promoting rhizobacteria (PGPR) are rootcolonizing bacteria able to provide growth and health benefits to plants through various mechanisms involving the expression of plant-beneficial functions (PBFs) in PGPR (Bruto et al. 2014; Lemanceau et al. 2017; Richardson et al. 2009; Vacheron et al. 2013). For example, PGPR can modulate plant hormonal signalization by producing phytohormones such as auxins, cytokinins, gibberellins, and nitric oxide (NO) (Bashan and de-Bashan 2010) or decreasing the emission of ethylene (Glick et al. 1998; Honma and Shimomura 1978; Vacheron et al. 2016a). PGPR may also help plant nutrition by solubilizing inorganic compounds, such as phosphate, or other mineral elements (Adesemoye and Kloepper 2009; Vessey 2003). Finally, some PGPR, in particular fluorescent pseudomonads, are producing antimicrobial compounds such as 2,4-diacetylphloroglucinol (DAPG), which protects plants against plant-pathogen microorganisms (Almario et al. 2014; Couillerot et al. 2009; Lanteigne et al. 2012) and activates plant defenses (Iavicoli et al. 2003). Some of these antimicrobial compounds can also act as a communication signal between bacteria (CombesMeynet et al. 2011).

The availability of an ever-increasing number of sequenced and annotated PGPR genomes is allowing us to perform some comparative genomic analyses (Flury et al. 2016; Loper et al. 2012). The results of these analyses show the existence of combinations of PBF genes that are specific to some PGPR taxa (Bruto et al. 2014; Vacheron et al. 2016b). For example, in strains or species belonging to the Pseudomonas fluorescens group, the genes required for the synthesis of DAPG are present simultaneously with the ones required for cyanid hydrogen (HCN) synthesis (Ramette et al. 2003). In Pseudomonas protegens, the production of pyoluteorin is cooccurring with the production of DAPG (Ramette et al. 2011). The presence of co-occurring PBF genes in genomes suggests that these functions might be part of common bacterial regulatory or metabolic networks. Alternatively, Bashan and de-Bashan (2010) hypothesized that each PBF present in one PGPR genome would improve plant growth either simultaneously or successively. In this additive hypothesis, the overall improvement of plant growth would result from the sum of effects of each co-occurring PBF. Although the relative contribution of several antimicrobial compounds to biocontrol performance has been well studied (Calderón et al. 2013; Iavicoli et al. 2003; Romanowski et al. 2011; Voisard et al. 1989), the contribution of co-occurring PBFs in plant growth promotion is poorly documented.

Among fluorescent pseudomonads, the Pseudomonas kilonensis F113 strain (formerly Pseudomonas fluorescens [Almario et al. 
2017]) is a PGPR strain isolated from sugar-beet rhizosphere (Shanahan et al. 1992). That strain is well-known for its biocontrol activity against a broad range of plant-pathogen microorganisms (Cronin et al. 1997a and b; Dunne et al. 1998). Less known, but all the more important, F113 also promotes plant growth and development, particularly on maize (Vacheron et al. 2016a; Walker et al. 2012), probably because of its rhizosphere competence or, in other words, its ability to efficiently colonize plant roots and to compete with other rhizosphere-competent bacteria (Lugtenberg and Dekkers 1999). The analysis of the F113 genome sequence reveals the presence of multiple PBFs (Redondo-Nieto et al. 2013). We can identify the phl genes, which enable the production of DAPG ( $p h l D$ encodes a polyketide synthase that is a key determinant of the DAPG biosynthesis) and, also, gcd encoding a glucose dehydrogenase involved in phosphate solubilization (Miller et al. 2010), acdS encoding the 1-aminocyclopropane-1-carboxylate (ACC) deaminase enzyme (Prigent-Combaret et al. 2008; Vacheron et al. 2016a), and nirS encoding the nitrite reductase involved in NO production (Choi et al. 2006). Specific associations of co-occurring PBFs were frequently observed among Proteobacteria (Bruto et al. 2014) and may reflect the existence of functional interactions occurring between PBFs. In F113, the interaction between the four PBFs (i.e., DAPG production, ACC deamination, phosphate solubilization, and NO production) may happen at a regulatory or metabolic level and could result, when combined all together, in enhanced plant growth. Following the additive hypothesis, we predict that the four co-occurring PBFs exert additive beneficial effects on the development of plant root-system architecture (RSA) and on the overall growth of plants by P. kilonensis F113. To test this hypothesis, we used a genetic approach and constructed five mutant strains: four deleted for each PBF and one with deletion of all four PBFs. These five mutants were used i) to analyze the functional relationships between F113 plantbeneficial activities and ii) to determine if they are all equally contributing to the plant beneficial effects of F113 on Arabidopsis thaliana in in vitro conditions and on maize (Zea mays L.) in a soil-pot experiment.

\section{RESULTS}

In vitro, most $P$. kilonensis F113 PBFs operate independently of each other.

The existence of functional interactions between the four cooccurring PBFs of P. kilonensis F113 was first tested in vitro and in absence of plants. In the wild-type F113 strain (WT) and in each various mutant strains (i.e., $\Delta a c d S, \Delta p h l D, \Delta n i r S, \Delta g c d$ and the quadruple mutant strain), we measured the activity of ACC deamination, phosphate solubilization, nitrite reduction and DAPG synthesis. As expected, the deamination of ACC into $\alpha$-ketobutyrate was impaired in the $\Delta a c d S$ and quadruple mutants (Fig. 1A). AcdS activity was weakly induced in $\Delta g c d$ and $\Delta$ nirS compared with F113 (1.7-fold and 1.3-fold, respectively) and not significantly affected in the $\triangle p h l D$ mutant (Fig. 1A). Moreover, the insoluble phosphate was no longer solubilized in the $\Delta g c d$ and quadruple mutants (Fig. 1B). Furthermore, the glucose dehydrogenase Gcd activity was weakly but significantly decreased (1.7-fold) in the $\Delta p h l D$ mutant but remained identical to the one of F113 in $\Delta$ nirS and $\Delta a c d S$ (Fig. 1B). The $\Delta$ nirS and the quadruple mutants were unable to reduce nitrite (and consequently to produce NO) (Fig. 1C). The NirS activity in the $\Delta a c d S$ or $\Delta g c d$ or $\Delta p h l D$ strains was identical to that of the F113 strain (Fig. 1C). As expected, there was no longer DAPG synthesis in the $\triangle p h l D$ and quadruple mutants (Fig. 1D). Interestingly, there was a strong accumulation of DAPG in the $\Delta g c d$ ( 82 times more than F113). By contrast the $\Delta a c d S$ and $\Delta$ nirS strains produced as much DAPG as did F113 (Fig. 1D). Thus, the main functional interaction that occurs in vitro between all four different PBFs is between the cellular pathways containing the glucose dehydrogenase Gcd and the polyketide synthase PhlD, in which both are involved in the control of DAPG biosynthesis in P. kilonensis F113.

To further study the interaction between DAPG synthesis and the glucose dehydrogenase, the DAPG production and phosphate solubilization were compared between all double and triple deletion mutants (Supplementary Fig. S1). On one hand, no phosphate solubilization was observed in all $\mathrm{gcd}$ double
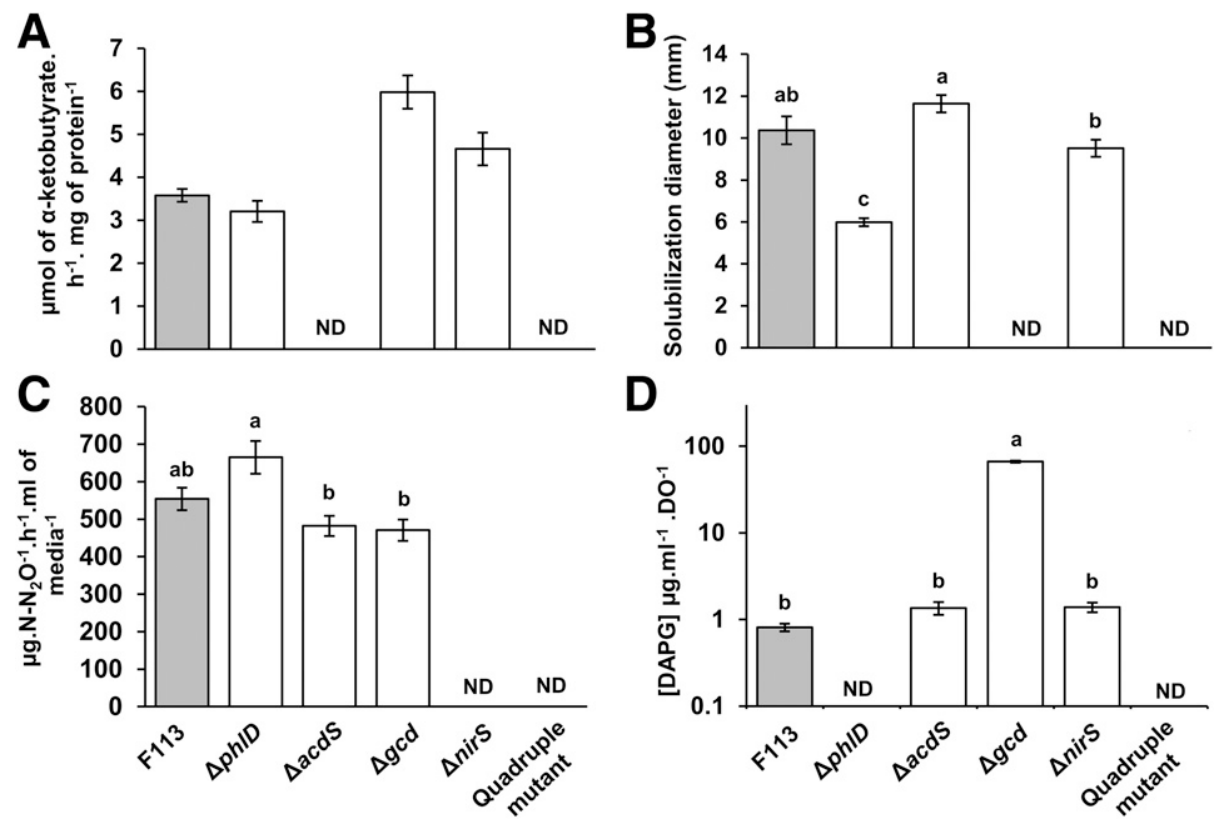

Fig. 1. Effect of the deletion of F113 plant beneficial functions (PBFs) on each PBF activity of the strain. A, 1-aminocyclopropane-1-carboxylate deaminase activity. B, Phosphate solubilization assay. C, $\mathrm{N}_{2} \mathrm{O}$ quantification. D, 2,4-diacetylphloroglucinol (DAPG) quantification. ND = nondetected. Statistical differences between strains for each tested PBF are indicated with letters (analysis of variance and Tukey's honestly significant difference test, $P<0.05$ ). Absence of letters indicates that differences were not significant. 
and triple mutants. It is also significantly reduced in the $\Delta p h l D \Delta a c d S$ (1.5-fold compared with F113), $\Delta p h l D \Delta$ nirS (1.3-fold compared with F113), and $\Delta p h l D \Delta a c d S \Delta$ nirS (1.7-fold compared with F113) mutants but in similar way than in the single $\Delta p h l D$ mutant (1.7-fold compared with F113). Thus, apart from the small positive impact of DAPG production on the solubilization of phosphate, there is no other function that interacts with the latter. On the other hand, DAPG production is impaired in all phlD double and triple mutants but is unaffected in a $\Delta a c d S \Delta$ nirS mutant. Compared with the increase observed in a single gcd mutant (82 times higher than F113), DAPG production is much more increased in a $\Delta a c d S \Delta$ gcd mutant (226 times higher than F113) but reduced in the $\Delta$ nirS $\Delta g c d$ (30 times higher than F113) and $\Delta a c d S \Delta g c d \Delta$ nirS mutants (26 times higher than F113). These results show that the cellular network involving the glucose dehydrogenase Gcd and the polyketide synthase PhlD is influenced negatively by acdS and positively by $\operatorname{nir} S$.

\section{Deletion of PBF genes does not affect the fitness of $P$. kilonensis F113 in vitro but impairs its rhizosphere soil competitiveness.}

When a PGPR does not enhance plant growth, it can be due to either the absence of a particular PBF in that strain, the poor expression of the PBF gene in the rhizosphere, or to a reduced rhizosphere competence. To assess if any of the tested PBFs can affect rhizosphere competence, we measured the growth of the WT strain and of every single and quadruple mutant strain in vitro and in vivo. In liquid culture, bacterial growth can be limited by the time required to divide (characterized by the growth rate) and the size of the cell population in the stationary phase (characterized by the final optical density [OD] reached in the liquid culture).

The growth rate of the quadruple mutant is higher than that of the WT or any given single mutant strain (Fig. 2; Supplementary Table S1). The growth rates of all simple mutants are similar to that of the WT (Fig. 2). In terms of final OD reached in stationary phase, the quadruple and the $\triangle p h l D$ mutants reached the highest OD in the stationary growth phase compared with any of the single mutants and with the WT strain (Fig. 2). Since neither the $\Delta p h l D$ nor the quadruple mutant synthesize DAPG in vitro (Fig. 1D), these results suggest that the production of DAPG might become toxic to F113 cells, at the stationary phase, in the tested conditions. To test that hypothesis, the growth of F113, the $\triangle p h l D$ mutant, and its complemented mutant was evaluated in the presence or absence of DAPG (Supplementary Fig. S2). First, the complemented $\Delta p h l D$ mutant had a growth kinetic curve similar to that of F113. Second, the addition of DAPG reduced significantly the OD in the stationary growth stage of F113 (starting from $0.1 \mu \mathrm{g}$ of DAPG per microliter). Third, the growth of $\triangle p h l D$ was slowed down in the exponential phase and was reduced in the stationary growth phase by the addition of DAPG at 0.5 and $1 \mu \mathrm{g} \mu \mathrm{l}^{-1}$. Fourth, the addition of DAPG (at 0.5 and $1 \mu \mathrm{g} \mu \mathrm{l}^{-1}$ ) slowed down the exponential growth stage of the complemented $\Delta p h l D$ mutant, but the OD reached in the stationary growth stage was quite similar to that of the complemented $\Delta p h l D$ mutant and of F113. Thus, when DAPG concentration reaches $0.5 \mu \mathrm{g} \mu \mathrm{l}^{-1}$, it negatively affects the growth of F113. Although the $g c d$ mutant produced much more DAPG than the WT (and more than approximately $0.51 \mu \mathrm{g} \mu \mathrm{l}^{-1}$ ), its growth is not affected. In any case, none of the simple and quadruple mutants have major deleterious effects on F113 cell growth allowing us to analyze their role during the interaction of F113 with plant.

To monitor the contribution of PBF genes in the fitness of F113 in maize rhizosphere, F113 and mutants were inoculated on maize seeds grown in natural soil, in the presence of the indigenous microbial communities. The same inoculation level of $5 \times 10^{6}$ bacteria per seed was used for each strain. F113 cell population was stable up to 8 days postinoculation (dpi) but decreased at 20 dpi (Fig. 3). At 8 dpi, all mutant strains were found to be less abundant than F113 in the rhizosphere, the $\triangle p h l D$ and quadruple mutants being the least numerous (Fig. 3). At $20 \mathrm{dpi}$, there was no significant difference in rhizosphere colonization between F113 and the $\Delta p h l D$ and $\Delta g c d$ mutants, whereas $\Delta a c d S, \Delta n i r S$, and the quadruple mutants were less abundant than F113 (Fig. 3). Thus, all PBFs contribute to F113 rhizosphere competence but to various extents. In particular, phlD is relevant for root colonization for up to 8 days and $a c d S$ and nirS genes are important for ensuring effective maize colonization over 20 days.

\section{The acdS, nirS, and gcd genes affect maize growth.}

F113 and PBF mutants were inoculated on maize seeds grown in pots filled with soil. Twenty days after, shoot and root architecture traits were measured, and the profiles obtained for each strain were compared using hierarchical clustering analyses

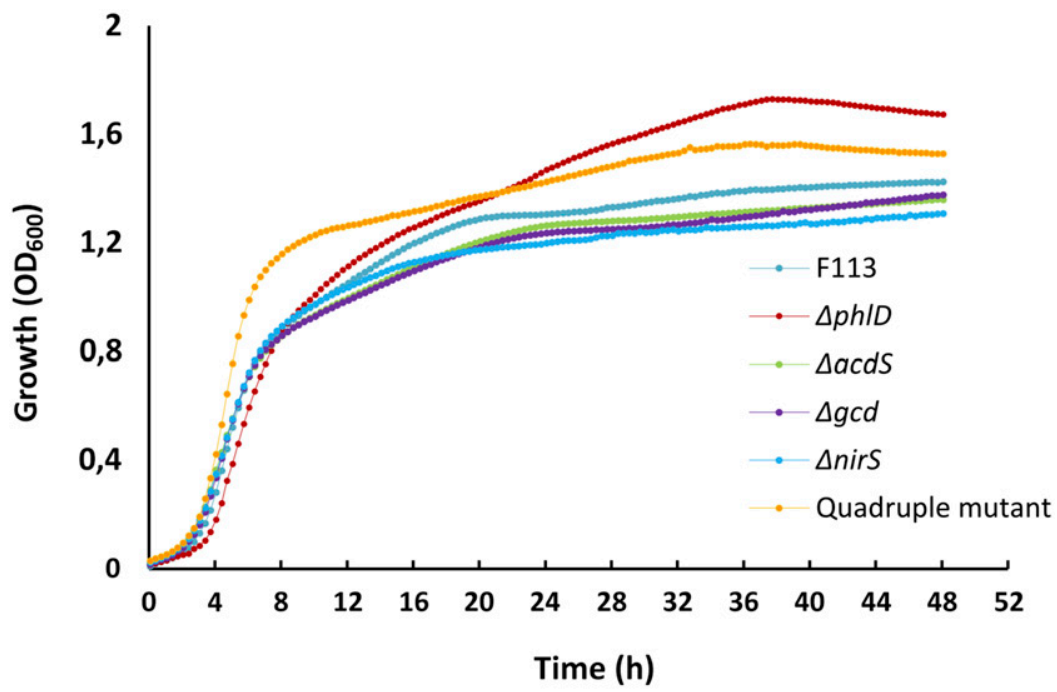

Fig. 2. Growth curve of Pseudomonas kilonensis F113 and its plant beneficial function mutants in King's B broth at $28^{\circ} \mathrm{C}$. The data represent the means of five replicate cultures (standard errors were not presented but were lower than $10 \%$ ). The experiment was done three times independently. 
(HCA). Compared with the noninoculated condition, F113 significantly enhanced root growth (with a $50 \%$ increase of total root length and $46 \%$ increase of total root surface) (Supplementary Fig. S2) and shoot growth (with an enhancement of the total leaf surface of 44\%) (Supplementary Table S3; Supplementary Fig. S3).

When comparing the inoculation effects of mutants to that of F113 on maize by HCA, two clusters were formed, F113, $\Delta p h l D$, and $\Delta g c d$ were grouped in one and $\Delta a c d S, \Delta n i r S$, and the quadruple mutant in the other (Fig. 4). In the first cluster, all the plant parameters appear evenly affected during inoculation, giving rise to a shape like a circle similar to the one obtained with F113. It suggests that there are either no differences or minor differences between F113 and the $\Delta p h l D$ and $\Delta g c d$ mutants. Consistent with that, there are no significant differences between F113 and $\triangle p h l D$ for all plant parameters assessed, while the inoculation with $\Delta g c d$ reduced significantly maize root length and surface with, respectively, 26 and $20 \%$ decrease compared with F113.

In the second cluster, the plant parameters appear to be unevenly affected, giving rise to an unsymmetrical shape. The three strains belonging to that cluster (i.e., $\Delta a c d S, \Delta$ nir $S$, and the quadruple mutant) have a similar impact on maize development and growth. Contrasting with the strains of the first cluster, the inoculation of $\Delta a c d S, \Delta n i r S$, and the quadruple mutant impacted the RSA significantly compared with that of F113, by reducing significantly the root length $(-64,-59$, and $-71 \%$, respectively), the root surface $(-50,-50$, and $-57 \%$, respectively), the number of root tips (respectively $-62,-45$, and $-71 \%$ ) and the root fresh weight (respectively, $-31,-38$, and $-42 \%$ ). Thus, the RSA modifications triggered by F113 on maize were lost when $\Delta a c d S, \Delta$ nirS or the quadruple mutants were inoculated.

In conclusion, F113 PBF genes and, in particular, acdS and nirS appear to alter mostly maize root parameters but not shoot parameters.
F113 PBFs differently affect $\boldsymbol{A}$. thaliana

root hair elongation and primary root growth in gnotobiotic agar conditions.

To further investigate F113 PBF impact on roots, we turned to in vitro experiments, as they allow a careful monitoring of F113 effects on root development or root hairs. Because maize is a large plant, with a root system that is likely to be too large to be evaluated in vitro, we turned instead to Arabidopsis, as its root development shares some similarities with that of maize (Atkinson et al. 2014; Hochholdinger and Zimmermann 2008).

We first verified the effect of F113 inoculation and that of the various mutant strains on Arabidopsis primary root growth. F113 inoculation inhibited significantly (twofold) the primary root length of $A$. thaliana (Fig. 5A). When A. thaliana Col-0 was inoculated with $\Delta p h l D$, a smaller reduction of the primary root growth (1.5-fold) compared with F113 was observed. However, the difference with F113 was not significant, implying that DAPG production only weakly contributed to the primary root length inhibition triggered by the WT strain (Fig. 5A). Contrarily, primary root length inhibition was lost when $\Delta a c d S, \Delta g c d, \Delta n i r S$, or the quadruple mutants were inoculated (Fig. 5A). Therefore, acdS, gcd, and nirS independently contribute to Arabidopsis primary root growth inhibition driven by F113 (Fig. 5A). Secondly, we investigated the effect of F113 and of the various mutant strains on the plant root-hair length. F113 inoculation led to significantly longer root hairs (Fig. 5B). Inoculation with the quadruple mutant generated the same plant phenotype as the noninoculated condition, suggesting that at least one of the deleted PBFs was responsible of enhanced root hair length (Fig. 5B). However, compared with F113 WTinoculated plants, the average root-hair lengths were significantly reduced by $20 \%$ in $\Delta p h l D-, 14 \%$ in $\Delta a c d S-, 21 \%$ in $\Delta g c d$-inoculated plants, and up $41 \%$ in $\Delta n i r S$-inoculated plants (Fig. 5B). Therefore, none of F113 PBF functions is solely controlling root-hair elongation. Because the activation of the plant ethylene signaling pathway results in root-growth inhibition

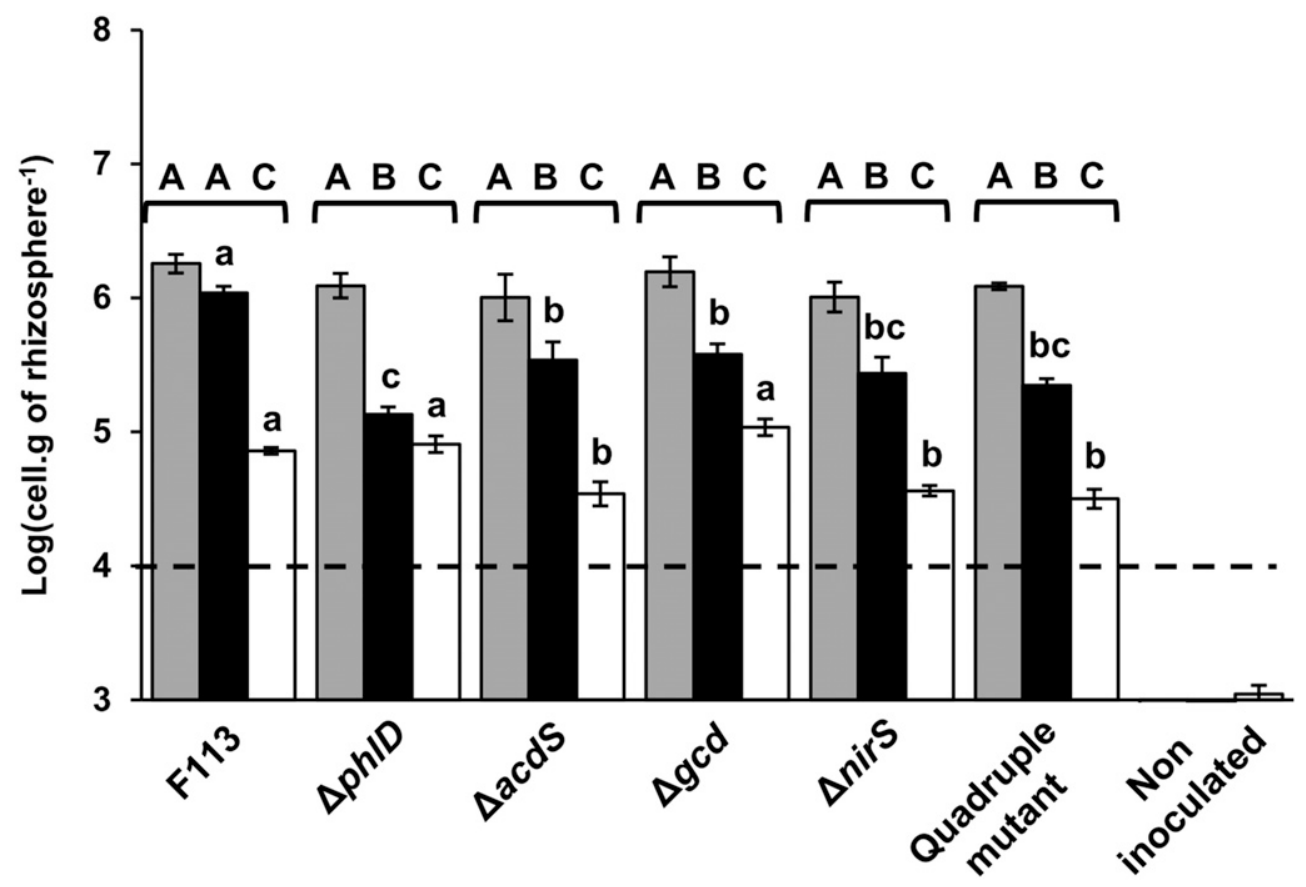

Fig. 3. Comparison of maize root colonization levels between Pseudomonas kilonensis F113 and its plant beneficial function mutants. The gray bar corresponds to root colonization at the moment of the seed inoculation (day 0), black at 8 days postinoculation (dpi), and white at 20 dpi. Statistical differences across the time are represented with upper case letters A to C. Statistical differences between strains at a given time are represented with lower case letters a to c. The dotted line corresponds to the quantitative polymerase chain reaction detection limit. 
and root-hair elongation (Pitts et al. 1998; Swarup et al. 2007) and is also an indicator of the activation of a defense-related mechanism (Broekgaarden et al. 2015), we looked at the effects of F113 and of the various mutant strains on plant ethylene emission by photoacoustic infrared spectrophotometry (Cristescu et al. 2013). Plant ethylene emissions increased significantly when F113 (1.5 fold) and $\Delta a c d S$ (twofold) were inoculated compared with the noninoculated plant. The plant ethylene emission produced by $\Delta p h l D, \Delta g c D, \Delta$ nirS, and the quadruple mutant did not significantly differ from that of the noninoculated condition (Fig. 6). In conclusion, all F113 PBFs are contributing to the production of ethylene by Arabidopsis; but in a distinct manner, acdS is reducing Arabidospis ethylene content whereas phlD, gcd, and nirS are increasing it.

\section{DISCUSSION}

In this work, we show that interactions between PBFs might be different when bacteria are growing in vitro or in contact with a plant, while functional overlapping between PBFs may occur by affecting the same plant pathway. It highlights that inactivation of one PBF gene can affect the activity of other ones and that all PBFs harbored by PGPR must be taken into account when analyzing the plant response effects of PGPR inoculation.

\section{Functional interactions}

between co-occurring PBFs in P. kilonensis F113.

In vitro, the main functional interaction occurring in F113 cells between the four studied PBFs is between phosphate solubilization and biosynthesis of DAPG. We showed that the glucose dehydrogenase activity (Gcd) is repressing DAPG biosynthesis in F113 (Fig. 1D), confirming observations formerly made in Pseudomonas protegens CHA0 (de Werra et al. 2009). In cells containing a functional Gcd, glucose-6P is transformed into gluconic acid, which is exported out of the cells, leading to medium acidification and phosphate solubilization (Stella and Halimi 2015). When gcd is deleted, a higher quantity of glucose-6P could be provided to the glycolysis, resulting, on the one hand, in increased ATP production by the cell and, on the other hand, in a higher amount of pyruvate that could then be incorporated to the tricarboxylic acid cycle to produce a higher amount of acetyl CoA, the precursor of DAPG (Bangera and Thomashow 1999; Clifford et al. 2016) (Supplementary Fig. S4). Interestingly, a metabolic interaction involving Gcd with another antimicrobial compound (i.e., pyoluteorin) has been evidenced in $P$. protegens CHA0 (de Werra et al. 2009).

DAPG was shown to act as an intracellular messenger controlling transcription of different PBF genes, such as pyoluteorin production in $P$. protegens $\mathrm{CHA0}$ and Pf-5 (Clifford et al. 2016; de Werra et al. 2011; Kidarsa et al. 2011; Schnider-Keel et al. 2000 ), or as an intercellular messenger inducing PBFs in other PGPR, as in auxin production (Combes-Meynet et al. 2011). Therefore, we hypothesize that the $\Delta g c d$ mutant strain could also show some specific modification at the transcriptional level of the expression of other PBF-encoding genes, resulting in potential functional compensation.

Distinct roles for F113 PBFs during colonization.

The functional importance of F113 PBF functions and its competitiveness against the native soil populations of rhizobacteria was tested on maize plants growing in pots filled with nonsterile soil, a setup close to natural conditions. For this experiment, we selected maize as a model because it is frequently grown in fields where the soil we used had been

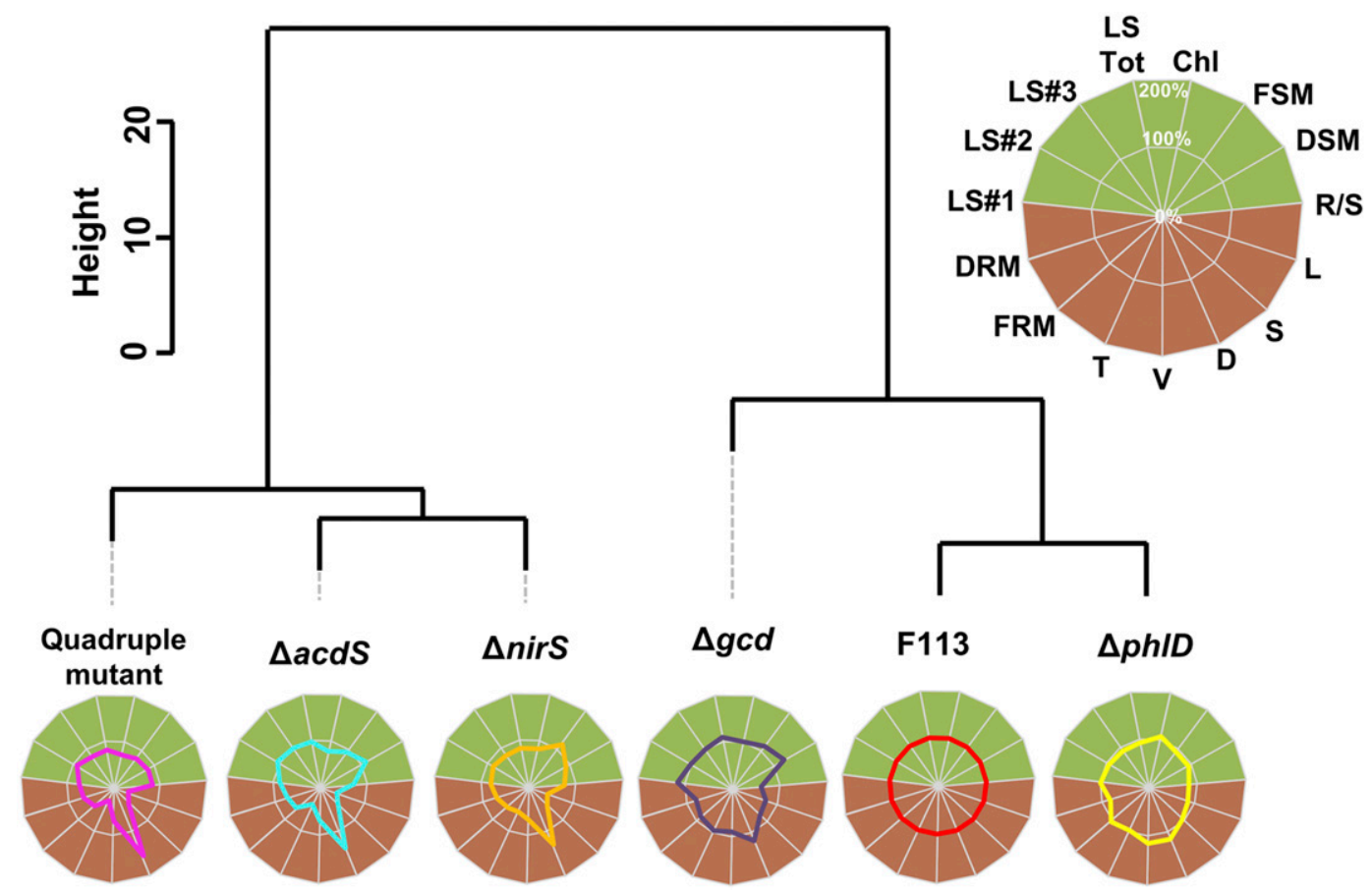

Fig. 4. Comparison of the effects of Pseudomonas kilonensis F113 plant beneficial function mutants on maize growth by hierarchical clustering. For each plant parameter, ratios between data obtained with mutants and with F113 were calculated and expressed as percentages. Root- and shoot-measured parameters are colored, respectively, in brown and green on the star plot. LS\#1, surface of leaf number 1 (same nomenclature for leaf 2 and 3); LSTot, total leaf surface; Chl, chlorophyll content in leaf number 3; FSM, fresh shoot mass; DSM, dry shoot mass; L, total root length; S, total root surface; D, root diameter; V, root volume; T, root tips; FRM, fresh root mass; DRM, dry root mass; R/S represents the ratio between the dry root biomass and the dry shoot biomass. Raw data were processed through hierarchical clustering analysis, using the Euclidean model and unweighted pair group method with arithmetic means as agglomeration method. 
collected, suggesting that this soil is enriched in a maizespecific rhizosphere microbiota that is ideal to compare the rhizosphere competitiveness of a mutant vs. a wild-type strain. In addition, maize plantlets have a large root system, allowing an easy quantification of the colonization process.

Root secretion of organic substances is essential for the rhizosphere microbiota to thrive (Haichar et al. 2016). Since the root exudates are composed of organic molecules, the origin of which can be traced back to photosynthetic activity of the plant, it is important that the two timepoints selected to sample the maize root rhizosphere will not differ in the physiological state of the plant. We sampled maize roots 8 and 20 days after sowing. In each case, we found the maize seedlings are already performing photosynthesis and may release root exudate whose composition is not drastically dissimilar. Consistent with this hypothesis, the composition in secondary metabolites present in root exudates from five distinct maize inbred lines is less different between 8 and 10 days than it is between 3 and 5 days postgermination (V. Walker and C. Prigent-Combaret unpublished results).

At $8 \mathrm{dpi}$, there are less $\Delta p h l D$ mutant bacteria in the maize root rhizosphere than F113 while, at 20 dpi, the $\Delta p h l D$ mutant strain is equally abundant as the WT (Fig. 3). This suggests that DAPG is important at the beginning of the colonization process. DAPG is a well-known antimicrobial compound secreted by F113 (Cronin et al. 1997a and b). Its secretion could limit the presence of competitor rhizobacteria or fungi in vivo, hence favoring an exclusive interaction between F113 and the host roots. Curiously, the $\Delta g c d$ mutant strain is equally abundant as the WT strain at both timepoints (Fig. 3). Yet, the $\Delta g c d$ mutant strain produces three times more DAPG in vitro than the WT strain (Fig. 1). Enhanced DAPG production may trigger some compensation mechanisms in the $\Delta g c d$ mutant strain, allowing it to colonize maize roots as efficiently as the WT.

At $20 \mathrm{dpi}$, the colonization of the $\Delta$ nirS, $\Delta a c d S$, and the quadruple mutant is less efficient than that of F113, $\Delta g c d$, and $\Delta p h l D$ (Fig. 3). AcdS ${ }^{+}$strains are catabolizing ACC exuded by plant roots in $\alpha$-ketobutyric acid (carbon source) and $\mathrm{NH}_{4}^{+}$ (nitrogen source) (Glick et al. 1998). acdS deletion can reduce the ability of F113 to fulfill its nutrient requirements in the rhizosphere, and thus, its fitness and root colonization ability. NirS is allowing bacteria of the Pseudomonas genus, a strictly respiring genus, to respire nitrite in the microaerophilic or anaerobic conditions that are frequently encountered in the rhizosphere (Højberg et al. 1999). Thus, the loss of nitrogen-respiring capacity may explain the lower root colonization ability of the $\Delta$ nirS mutant that was previously observed (Ghiglione et al. 2002) as well as in our condition (Fig. 3). Since both NirS and AcdS are

A

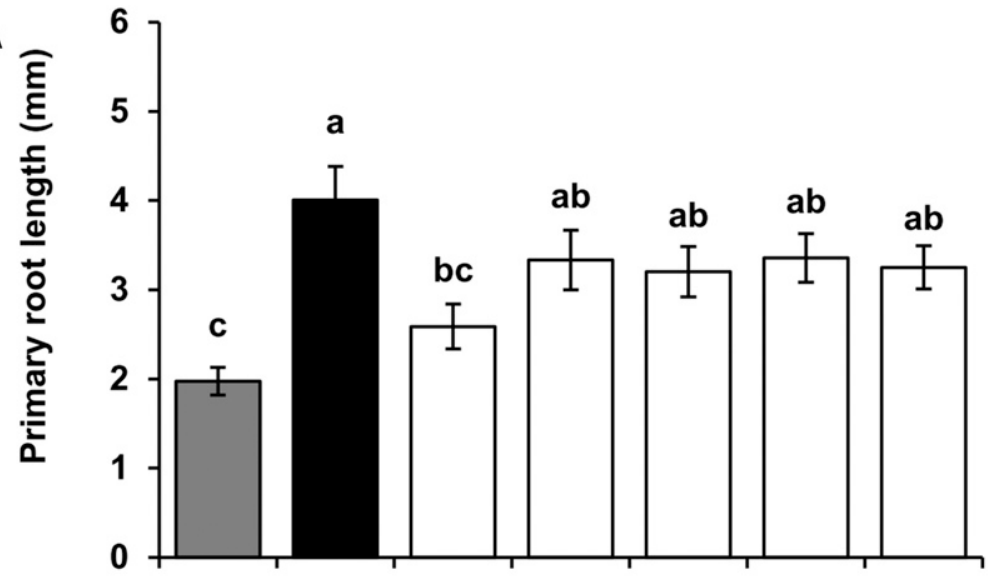

B

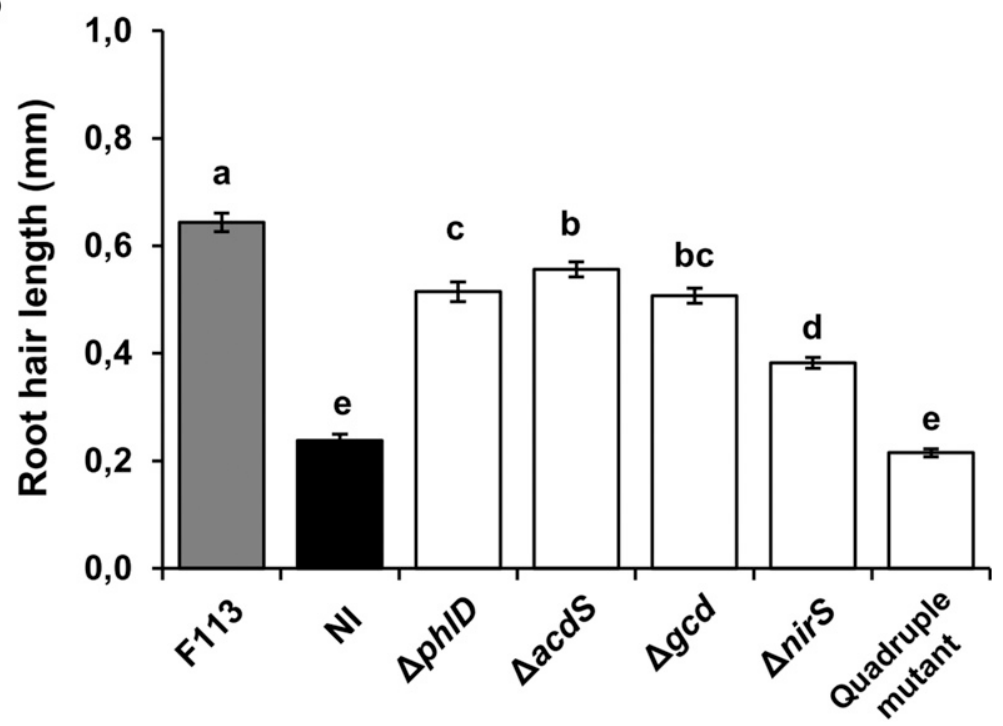

Fig. 5. Effects of the inoculation of Pseudomonas kilonensis F113 and its plant beneficial function mutants on the length of A, Arabidopsis primary roots and B, root hairs. NI = noninoculated condition. Conditions harboring the same letter were not significantly different (analysis of variance and Tukey's honestly significant difference test, $P<0.05$ ). 
important for the colonization at $20 \mathrm{dpi}$, one could have expected that in the quadruple mutant strain, the effects of the two mutations would add up, resulting in a strain with very poor ability to colonize at 20 dpi. Surprisingly, this is not the case (Fig. 3). This suggests the existence of potential epistatic interactions between the two genes nirS and $a c d S$ or with one or both $g c d$ and phlD that our in vitro analysis failed to unravel (Fig. 1). It also might be that plant exudates or microbe-microbe interactions in the rhizosphere result in activation of other genes that compensate for the lack of nirS and $a c d S$.

\section{The maize colonization process by $\mathrm{F} 113$ has} a significant impact on plant root and shoot growth.

To test if these different colonization patterns could affect maize growth in vivo, we measured 15 different physiological parameters (eight for shoots and seven for roots). To get a broad view of the effect of $\mathrm{F} 113$ on maize plants, we have used a hierarchical clustering analysis (HCA). Instead of focusing on each parameter, HCA considers parameter patterns that can be compared between the mutant and the WT strains (Fig. 4). The inoculation of maize with the $\Delta p h l D$ or $\Delta g c d$ strains resulted in a pattern similar to that of F113 (Fig. 4). Since phlD appears to play a role for maize root colonization at 8 dpi (Fig. 3), it suggests that F113 colonization of maize roots after 8 dpi is more important for the effects on maize growth than up to $8 \mathrm{dpi}$. Consistent with that interpretation, the inoculation of maize roots with the $\Delta \operatorname{nirS}$ or $\Delta a c d S$ or the quadruple mutant strain (all these genes are important for colonization at 20 dpi [Fig. 3]) results in a pattern different from that of the WT (Fig. 4).

Contrasting with F113, inoculation with $\triangle a c d S$ or $\Delta$ nirS or the quadruple mutant resulted in a decrease in total root length, total root surface, and number of root tips and, at the same time, an increase in root diameter (Fig. 4). This indicates that acdS and nirS, two genes that are important for the fitness of F113, are also important for root growth promotion. How they could be beneficial to root maize growth remains unclear. For example, it could be a direct interference with the plant ethylene pathway for AcdS or with the plant NO pathways for NirS. At the level of the shoot, maize plants inoculated with $\Delta a c d S$ or $\Delta n i r S$ or the quadruple mutant show a weak decrease in leaf surfaces as well as in chlorophyll content, compared with F113 (Fig. 4). While the leaf surface of maize plants inoculated with
$\Delta a c d S, \Delta$ nir $S$, or the quadruple mutant are slightly higher than in the noninoculated plants, the chlorophyll content is lower. As chlorophyll fluorescence is reduced in stressed plants (BolhàrNordenkampf and Öquist 1993), this result could suggest that maize plants inoculated with $\Delta a c d S$ or $\Delta$ nirS or the quadruple mutant are stressed, whereas F113 may alleviate plant stress. Taken together, the PBF functions that contribute to the longterm fitness of F113 are also the ones that have the strongest effects on the growth of maize plants.

\section{Relative contribution of PBFs on $A$. thaliana root architecture.}

To further study the contribution of the F113 various PBFs on root development, we performed in vitro experiments with plants. We have used Arabidopsis as a model plant because it is small, grows fast, has a relatively simple root development program (tap root), and its root hairs are very easily observable. In addition, maize and Arabidopsis root development programs share some similarities (Atkinson et al. 2014; Hochholdinger and Zimmermann 2008). Finally, F113, which was initially isolated from sugar beet roots, is known to colonize efficiently both monocotyledons such as wheat (Couillerot et al. 2011; De La Fuente et al. 2006) and maize (Couillerot et al. 2013; Von Felten et al. 2010) but, also, dicotyledonous crops such as alfalfa (Sánchez-Contreras et al. 2002; Villacieros et al. 2003) or tomato (de Weert et al. 2002).

In vitro F113 inoculation resulted in Arabidopsis primary root growth inhibition and strong promotion of root hair elongation (Fig 5A and $\mathrm{B}$ ). By contrast, the quadruple mutant showed no root architecture difference compared with the noninoculated Arabidopsis, suggesting that at least one F113 PBF or a combination of PBFs may trigger root architecture modifications (Fig. 5). Several plant hormones, such as auxin (controlling cell division and elongation) (Spaepen and Vanderleyden 2011) or ethylene (controlling root cell elongation) (Jung and McCouch 2013; Street et al. 2015; Swarup et al. 2007), control primary root elongation. However, F113 does not produce auxinic compounds (J. Vacheron, D. Muller, and C. Prigent-Combaret unpublished results) but, instead, produces DAPG (Fig. 1). One possibility is that DAPG may have an effect on the plant root system. Consistent with this, when tomato seedlings are grown in vitro and in the presence of $10 \mu \mathrm{M}$ DAPG, their

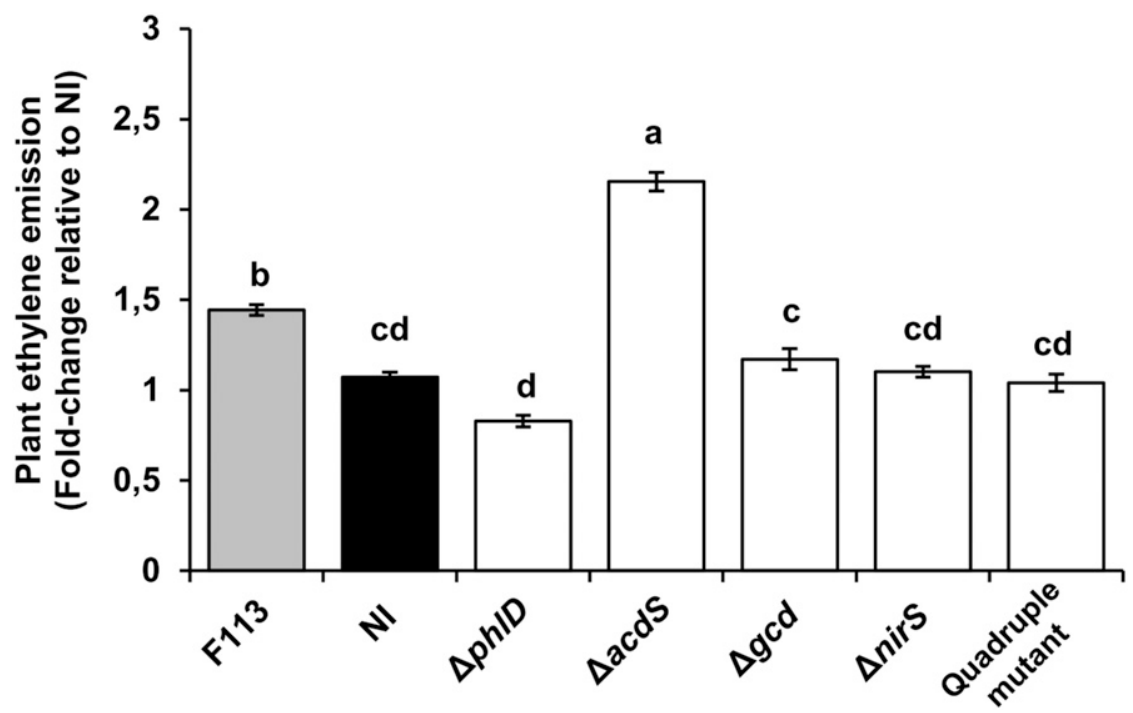

Fig. 6. Plant ethylene emission in response to the inoculation of Arabidopsis plantlets with Pseudomonas kilonensis F113 and its plant beneficial function mutants. Plant ethylene emission was calculated related to the plant ethylene emission for the noninoculated condition (NI). Conditions harboring the same letter were not significantly different (analysis of variance and Tukey's honestly significant difference test, $P<0.05$ ). 
primary root growth is inhibited (Brazelton et al. 2008). However, the $\triangle p h l D$ mutant (which does not produce DAPG) remains able to inhibit Arabidopsis primary root elongation, and the $\Delta g c d$ mutant (which overproduces DAPG) does not show further reduced primary root length compared with F113 (Fig. 5). In addition, both the $\Delta p h l D$ and $\Delta g c d$ mutants remain able to stimulate root hair length. These results imply that, aside from DAPG, other F113 PBFs are involved in the inhibition of primary root elongation and stimulation of root hair length in A. thaliana. Interestingly, the loss-of-function mutation in nirS significantly affects the inhibition of primary root growth (Fig. 5A) and root hair elongation (Fig. 5B). This may suggest that NO, produced by F113 NirS activity, as evidenced by using the specific fluorescent probe diaminorhodamine-4M acetoxymethyl ester (J. Vacheron, D. Muller, and C. PrigentCombaret unpublished results), would act as an inhibitor of primary root growth and activator of root hair elongation. Consistent with this, NO is described as a positive regulator of root hair development (Lombardo and Lamattina 2012; Lombardo et al. 2006) and a negative regulator of primary root growth (FernándezMarcos et al. 2011).

In the absence of PGPR, plant ethylene is known to inhibit primary root length and to activate root hair elongation (Cao et al. 1999; Pitts et al. 1998). F113 moderately increased plant ethylene emission, whereas all mutants but $\Delta a c d S$ shared similar plant-ethylene emission as in noninoculated conditions (Fig. 6). Individually, each of the F113 PBFs contributes positively to ethylene production by the plant during the associative symbiosis with $A$. thaliana (Fig. 6). Contrasting with the other mutants, the $\Delta a c d S$ strain colonization results in higher ethylene emission by the plant (Fig. 6). Hence, the AcdS activity negatively contributes to ethylene production. In our study, this mutant strain is the only simple mutant unable to degrade in vitro the ethylene precursor ACC (Fig. 1A). Assuming that the mutant strain behaves similarly in vitro and in vivo, the lack of AcdS activity results in higher ethylene emission by the plant (Fig. 6). However, this increase is not observed with the inoculation of the quadruple mutant, showing that the other PBFs activate plant ethylene production. Thus, it confirms that F113 colonization of the root system results in modulation of ethylene emission by the plant and that AcdS is not the only F113 PBF contributing to this effect. However, it remains to be seen if this emission is greater in the root, in the shoot, or both plant organs. It also confirms that AcdS activity is actively decreasing plant ethylene emission during the associative symbiosis. This result is consistent with a longstanding hypothesis on the function of AcdS during the interaction of AcdS $S^{+}$bacteria with plants (Glick et al. 1998). Yet, this increased emission of ethylene in the presence of the $\Delta a c d S$ mutant strain does not result in a shorter primary and longer root hairs compared with the effect of the WT strain (Fig. 5A and B). This absence of stronger root responses contrasts with previous observations suggesting that an absence of AcdS activity resulted in longer root hairs (Galland et al. 2012). But Phyllobacterium brassicacearum STM196, Rhizobium leguminosarum 128C53K, and Pseudomonas sp. strain UW4, the strains used in that previous work, may not have the same combination of PBFs as that of F113. Therefore, one should not rule out the possibility that this absence of stronger root responses with the $\Delta a c d S$ strain is specific to F113 and its combination of PBFs. Another possibility that could explain the absence of stronger root responses, despite a significant increase in ethylene emission, is that F113 $\Delta a c d S$-inoculated plants are becoming insensitive to the effect of high ethylene on root cells.

Taken together, F113 may trigger both ethylene- and NOdependent signaling pathways on Arabidospsis. Contrasting with the observations made on soil pot-grown maize, all F113s
PBFs tested in vitro on Arabidopsis contribute to modification of the root architecture.

\section{MATERIALS AND METHODS}

Bacterial strains, plasmids, and culture conditions.

The bacterial strains and plasmids used in this study are listed in Supplementary Table S4. P. kilonensis F113 WT and its mutants were incubated at $28^{\circ} \mathrm{C}$ in King's B broth media (Simon and Ridge 1974) supplemented, when required, with gentamycin $\left(15 \mu \mathrm{g} \mathrm{ml}^{-1}\right)$ or kanamycin $(\mathrm{Km})\left(50 \mu \mathrm{g} \mathrm{ml}^{-1}\right)$. Escherichia coli was incubated at $37^{\circ} \mathrm{C}$ in Luria Bertani broth (Bertani 1951) supplemented with gentamycin $\left(15 \mu \mathrm{g} \mathrm{m}{ }^{-1}\right)$ or ampicillin $\left(75 \mu \mathrm{g} \mathrm{ml}^{-1}\right)$ and $\mathrm{Km}\left(50 \mu \mathrm{g} \mathrm{ml}^{-1}\right)$, when necessary. To assess the effects of DAPG on the growth of F113 and its mutants, DAPG (Santa Cruz Biotechnology) was added in King's B broth medium at three different concentrations $\left(0.1,0.5\right.$, and $\left.1 \mathrm{mg} \mathrm{ml}^{-1}\right)$.

\section{Growth kinetic assays.}

Bacterial growth was monitored for $24 \mathrm{~h}$ at $28^{\circ} \mathrm{C}$ by measuring $\mathrm{OD}$ in a Bioscreen $\mathrm{C}$ analyzer (Labsystems) measuring the $\mathrm{OD}_{600}$ every $15 \mathrm{~min}$. Growth kinetic assays were performed three times independently with five technical repetitions.

\section{Construction of deletion mutants and complementation of the phlD deletion mutant.}

To construct F113 PBF deletion mutants, about 700-pb fragments were amplified from regions upstream and downstream from the PBF genes of interest, using specific primers (Supplementary Table S5), and fragments were subcloned into pGEM-T Easy (Promega). The upstream and downstream DNA fragments were digested with AatII/MunI and SacII/SacI for $\Delta p h l D$ and $\Delta g c d$, AatII/PvuII and SacII/SacI for $\Delta a c d S$, $P v u \mathrm{II} / \mathrm{MunI}$ and $\mathrm{SacII} / \mathrm{SacI}$ for $\Delta$ nirS and were then cloned, one after another, into pCM184, on both sides of the Km resistanceencoding gene (Marx and Lidstrom 2002). All restriction enzymes were supplied by ThermoFisher Scientific. All plasmid amplifications were done using E. coli JM109 chemically competent cells (Promega). For validation of the correct insertion of upstream and downstream DNA fragments into pCM184, sequencing, using primers $\mathrm{S} 1 \mathrm{~F} / \mathrm{S} 1 \mathrm{R}$ and $\mathrm{S} 2 \mathrm{~F} / \mathrm{S} 2 \mathrm{R}$, was performed. pCM184 harboring upstream and downstream regions of the plant-beneficial genes of interest were transformed into F113 by electroporation (Højberg et al. 1999). Inactivation mutants resulting from a double recombination event of upstream and downstream regions were selected on King's B Km and were screened for plant-beneficial gene deletion by polymerase chain reaction (PCR )with specific primers. Then, the plasmid CM157 containing the cre-lox recombinase gene was introduced into F113 Km-resistant mutants in order to remove the antibiotic marker. To check the loss of the Km cassette, mutant strains were screened for their inability to grow on King's B Km. PCR using external primers was also used to validate the cassette loss. For complementation of the phlD deletion mutant, the coding region of the phlD gene of F113 was amplified by PCR, using primers phlDCompF1 and phlDCompR1, and was subcloned into pGEM-T Easy. The phlD gene was extracted from pGEM-T Easy using ApaI/SacI digestion and was inserted into the multiple cloning site of vector pBBR1MCS-5, under control of the lac promoter, resulting in pBBR1-MCS5:phlD. This plasmid was introduced into the $\triangle p h l D$ mutant by electroporation (Højberg et al. 1999).

\section{DAPG quantification}

by high-pressure liquid chromatography (HPLC).

DAPG production was measured by HPLC, according to the method developed by Bonsall et al. (1997). Briefly, samples 
were analyzed with an Agilent 1200 series HPLC (Agilent Technologies) equipped with a degasser (G132A), a quaternary pump module (G1311A), an automatic sampler (G1329A), and a diode array detector (DAD G1315B). Separation of compounds was performed at room temperature with a $\mathrm{C} 18$ reversephase column. For each sample, $20 \mu \mathrm{l}$ was injected and eluted at $1 \mathrm{ml} \mathrm{min}{ }^{-1}$, using a step-by-step gradient increasing the acetonitrile $(\mathrm{ACN})$ proportion in water; the gradient started at $40 \%$ of $\mathrm{ACN}$ for $4 \mathrm{~min}$ then, rose from 40 to $64 \%$ in $7.5 \mathrm{~min}$, then came to $75 \%$ at $16.5 \mathrm{~min}$, and ended at $100 \%$ at $18.5 \mathrm{~min}(100 \%$ maintained for $3 \mathrm{~min}$ before decreasing to $40 \%$ for $5 \mathrm{~min}$ ). Chromatograms were recorded at $270 \mathrm{~nm}$ (maximum of absorbance of DAPG). The chemstation Agilent software was used for integration of chromatograms, and quantitation of DAPG was done, according to a reference curve, with the chemical standard (Sigma-Aldrich). This experiment was done independently three times.

\section{ACC deaminase activity assay.}

The ACC deaminase activity of strains was performed as described by Blaha et al. (2006). The method is based on the quantification of $\alpha$-ketobutyrate produced by the deamination of ACC into $\alpha$-ketobutyrate by the enzyme ACC deaminase. Total protein content in the assay was measured using the BCA protein assay kit (Pierce). ACC deaminase activity was assessed three times for each strain. The data are expressed in nanomoles of ketobutyrate per hour per milligram of protein. This experiment was done independently three times.

\section{Phosphate-solubilizing assay.}

The phosphate-solubilizing ability of strains was evaluated by measuring tricalcium phosphate solubilization halos on National Botanical Research Institute's Phosphate (NBRIP) agar medium. Strains were cultured for 1 day at $28^{\circ} \mathrm{C}$ in King's $\mathrm{B}$ broth and, then, were deposited four times ( $5 \mu \mathrm{l}$ each) on NBRIP agar medium. Solubilization halos were measured after 6 days of incubation at $28^{\circ} \mathrm{C}$, using ImageJ software. This experiment was done three times independently.

\section{Denitrification activity measurement.}

The denitrification activity was estimated through the potential accumulation of $\mathrm{N}_{2} \mathrm{O}$ in the culture media, which is one step of the denitrification process. The accumulation of $\mathrm{N}_{2} \mathrm{O}$ was measured according to Bardon et al. (2016). Briefly, $20 \mathrm{ml}$ of King's B media supplemented with $20 \mathrm{mM}$ of $\mathrm{KNO}_{3}$ were placed in a plasma flask sealed by a rubber stopper. An anaerobic condition was obtained by removing air and replacing it with a $\mathrm{He} / \mathrm{C}_{2} \mathrm{H}_{2}$ mixture (90:10, vol/vol) in order to inhibit the reduction of $\mathrm{N}_{2} \mathrm{O}$ to $\mathrm{N}_{2}$ and to allow $\mathrm{N}_{2} \mathrm{O}$ accumulation. Each strain was inoculated at a final $\mathrm{OD}_{600}$ of $0.1 . \mathrm{N}_{2} \mathrm{O}$ emissions were measured every $2 \mathrm{~h}$ for $24 \mathrm{~h}$, using gas chromatography coupled to a microkatharometer detector ( $\mu$ GC-R3000; SRA Instruments). The assay was performed twice with two technical replicates. $\mathrm{N}_{2} \mathrm{O}$ emission was expressed as micrograms of $\mathrm{N}-\mathrm{N}_{2} \mathrm{O}$ per hour per milliliter of media. In addition, the ability of F113 to produce NO intracellularly was evaluated, using the specific fluorescent probe diaminorhodamine-4M acetoxymethyl ester (Calbiochem), as previously described by Pothier et al. (2007).

\section{Bacterial inoculum preparation.}

P. kilonensis F113 WT and related deletion mutants $\Delta p h l D, \Delta a c d S$, $\Delta g c d, \Delta n i r S$, and the quadruple mutant $(\Delta p h l D \Delta a c d S \Delta g c d \Delta n i r S)$ were incubated overnight at $28^{\circ} \mathrm{C}$ in King's B broth medium. Cell cultures were centrifuged at 5,000 $\times g$. The pellet was washed with sterilized water and was adjusted to $10^{7}$ bacteria per milliliter.

\section{Bacterial inoculation effects on Arabidopsis RSA.}

The seeds of Arabidopsis thaliana ecotype Columbia 0 (Col-0) were prepared according to Mantelin et al. (2006). After 7 days, plantlets were transferred to $50 \mathrm{ml}$ of new plant agar medium containing F113 or mutant strains at $10^{7}$ cells per milliliter in square Petri dishes $(120 \times 120 \mathrm{~mm})$. Inoculated plantlets were transferred to a phytotron set to the following parameters: $21^{\circ} \mathrm{C}$ day and $17^{\circ} \mathrm{C}$ night temperatures, 13 -h light periods, 9,500 lux. Four Arabidopsis plantlets were disposed per Petri dish and six plates were prepared per condition. The seven conditions tested were A. thaliana inoculated with F113, with the four simple mutants, with the quadruple mutant, and the nonbacterized control. This experiment was made three times independently. After 7 days, three plants per Petri dish were scanned, in order to analyze the primary root elongation and root hair length, using ImageJ software (i.e., 18 analyzed plants per condition). The relative contribution of each tested PBF in the modulation of the Arabidopsis root architecture was calculated as follows.

$$
\begin{gathered}
\mathrm{F} 113-\text { control }=\alpha \\
{[(\mathrm{F} 113-\text { mutant }) / \alpha] \times 100=} \\
\text { deleted gene relative } \\
\text { contribution to } \mathrm{F} 113 \operatorname{effect}(\%)
\end{gathered}
$$

\section{Arabidopsis ethylene emission.}

Measurements of in vivo ethylene emission by Arabidopsis plantlets were performed using infrared laser spectroscopy coupled to photo-acoustic detection (Cristescu et al. 2013) (ETD-300, SensorSense). Practically, we carefully poured $600 \mu \mathrm{l}$ of sterile plant culture medium (as described above), inoculated or not with the various F113 strains at a final density of $2 \times 10^{7}$ cells per milliliter, in a 2-ml sterile Eppendorf tube. Once solidified, we carefully introduced two wild-type 7-dayold Arabidopsis plantlets and closed the lid. Lids were punched to allow for regular breathing of the plants. This system was then transferred to a phytotron (MLR-352-PE, Panasonic) set to the following parameters: $21^{\circ} \mathrm{C}$ day and $17^{\circ} \mathrm{C}$ night temperatures, a 13-h light period, 9,500 lux. No measure was made during the first $48 \mathrm{~h}$, to allow plant acclimation and to recover from stress associated to the transfer. Prior ethylene emission measurement, $200 \mu \mathrm{l}$ of plant liquid medium was added in each tube to keep the head space humid and, therefore, protecting the plant from the dry air coming from the compressed air bottle. Then, the lids of the tubes were removed and replaced by a rubber plug. Needles were pushed through the plug to allow the headspace to be flushed to the ethylene detector by compressed air. Every $90 \mathrm{~min}$, the ethylene content in the headspace was measured while the tubes remained in the phytotron. That measurement was performed during two to three consecutive days, to collect enough data on ethylene content during the day. At the end of the experiment, plant fresh weight was measured to allow data normalization. Raw results were exported into a spreadsheet, in which they were normalized and processed to obtain at least 10 different measures of ethylene content in the presence of light in each condition.

\section{Maize inoculation experiments in nonsterile soil.}

The semilate maize hybrid PR37Y15 (Pioneer Semences) was used in this study. Maize seeds were surface-disinfected. Briefly, seeds were soaked in sodium hypochlorite $(3.4 \%)$ for $1 \mathrm{~h}$, with agitation. Five washes were performed with sterile water. Four maize seeds were sown in nonsterile soil taken from La Côte Saint-André near Lyon, France (Vacheron et al. 2016a) and were placed in $2-\mathrm{dm}^{3}$ pots filled with $2.0 \mathrm{~kg}$ of sieved soil $(5 \mathrm{~mm})$ with a water content of $20 \%$ ( $\mathrm{vol} / \mathrm{vol})$. Moisture was 
maintained at $20 \%$ during the experiment. Seeds (four per pot) were inoculated with $100 \mu \mathrm{l}$ of bacterial inoculum solution, corresponding to $10^{6}$ bacteria. Ten pots per condition were used. Inoculum impact on plant growth was assessed at 20 dpi by characterizing maize RSA, using WinRhizo (Reagent Instruments). Leaf surface was measured with the WinFolia image analysis software (Regent Instruments). Chlorophyll content was measured with a chlorophyll content meter (Hansatech Instruments model CL-01). Root and shoot fresh and dry biomasses were weighed. Ten plants were analyzed per condition.

\section{Monitoring the bacterial maize root colonization.}

The measurement of root colonization was assessed at 0,8 , and $20 \mathrm{dpi}$, taking 10 seeds for each condition at each sampling time. Inoculated seeds or plants were harvested and disposed in a 50-ml Falcon tube and were then soaked in liquid nitrogen. Seeds and root systems were lyophilized and, then, were scratched to extract DNA from the seed surface and rootadhering soil. DNA extractions from $500 \mathrm{mg}$ of rhizosphere soil or seed teguments were performed using FastDNA SPIN Kit for Soil (MP Biomedicals). DNA was also extracted from $1 \mathrm{ml}$ of inoculum solution, to ascertain the cell concentration of the inoculants. The seed and root bacterial colonization was assessed by real-time PCR as described by Von Felten et al. (2010). Real-time PCR was performed using the LightCycler FastStart DNA Master SYBR Green I kit and a LC-480 LightCycler (Roche Applied Science). Five nanograms of extracted DNA were used to assess the abundance of seed and root colonization by the F113 parental and mutant strains, using F113_1_for and F113_1_rev primers with a detection limit of $10^{4}$ cells per seed and $10^{4}$ cells per gram of rhizosphere (Von Felten et al. 2010). Real-time PCR quantification data were converted to gene copy number per gram of lyophilized root, as described by Couillerot et al. (2013).

\section{Statistical analysis.}

Regarding assays on each plant-beneficial function, data were log-transformed, to respect normal distribution, and variances homogeneity and a one-way analysis of variance (ANOVA) and Tukey's honestly significant difference (HSD) tests were performed to detect significant differences between strains. Arabidopsis root hair length data were compared, using Kruskal-Wallis tests followed by posthoc nonparametric multiple comparisons. Arabidopsis primary root length data were square root-transformed in order to respect variance homogeneity and normality and a one-way ANOVA coupled with Tukey's HSD tests was performed. Ethylene emission data were expressed related to noninoculated condition and were compared, using ANOVA coupled with Tukey's HSD tests. The comparison of root colonization between strains at the same sampling time was analyzed by ANOVA and Tukey's HSD tests. The comparison of root colonization across the time for each strain was analyzed by ANOVA and Fisher's least significant difference tests. The effects of strain inoculation on maize growth parameters were compared, using ANOVA and Tukey's HSD tests and were represented using star plot representation coupled to hierarchical clustering analysis performed on the raw data (distance model: Euclidean; agglomeration method used: unweighted pair group method with arithmetic means). All data were presented as mean \pm standard error. All analyses were performed at $P<0.05$, using $\mathrm{R}$ studio software (R Studio Team 2015).

\section{ACKNOWLEDGMENTS}

We thank M. Ribierre for technical help and S. Puijalon and V. Gardette from UMR CNRS 5023 for allowing us to use the Winfolia software.
J. Vacheron was supported by a Ph.D. grant from Academic Research Cluster 3 of Rhône-Alpes Region. This work made use of the 'Serre' technical platform of FR41 at Université Lyon 1 and the AME plateform (UMR 5557) for denitrification assays. We also thank D. Aymoz for English proofreading of this paper. This study was supported by the ANR project SymbioMaize (ANR-12-JSV7-0014-01).

\section{LITERATURE CITED}

Adesemoye, A. O., and Kloepper, J. W. 2009. Plant-microbes interactions in enhanced fertilizer-use efficiency. Appl. Microbiol. Biotechnol. 85:1-12.

Almario, J., Bruto, M., Vacheron, J., Prigent-Combaret, C., MoënneLoccoz, Y., and Muller, D. 2017. Distribution of 2, 4-diacetylphloroglucinol biosynthetic genes among the Pseudomonas spp. reveals unexpected polyphyletism. Front. Microbiol. 8:1218.

Almario, J., Muller, D., Défago, G., and Moënne-Loccoz, Y. 2014. Rhizosphere ecology and phytoprotection in soils naturally suppressive to Thielaviopsis black root rot of tobacco. Environ. Microbiol. 16: 1949-1960.

Atkinson, J. A., Rasmussen, A., Traini, R., Voß, U., Sturrock, C., Mooney, S. J., Wells, D. M., and Bennett, M. J. 2014. Branching out in roots: Uncovering form, function, and regulation. Plant Physiol. 166:538-550.

Bangera, M. G., and Thomashow, L. S. 1999. Identification and characterization of a gene cluster for synthesis of the polyketide antibiotic 2,4diacetylphloroglucinol from Pseudomonas fluorescens Q2-87. J. Bacteriol. 181:3155-3163.

Bardon, C., Piola, F., Haichar, F. Z., Meiffren, G., Comte, G., Missery, B., Balby, M., and Poly, F. 2016. Identification of B-type procyanidins in Fallopia spp. involved in biological denitrification inhibition. Environ. Microbiol. 18:644-655.

Bashan, Y., and de-Bashan, L. E. 2010. Chapter two. How the plant growthpromoting bacterium Azospirillum promotes plant growth-A critical assessment. Pages 77-136 in: Advances in Agronomy. Academic Press, New York.

Bertani, G. 1951. Studies on lyzogeny I. The mode of phage liberation by lysogenic Escherichia coli. J. Bacteriol. 62:293-300.

Blaha, D., Prigent-Combaret, C., Mirza, M. S., and Moënne-Loccoz, Y. 2006. Phylogeny of the 1-aminocyclopropane-1-carboxylic acid deaminaseencoding gene $a c d S$ in phytobeneficial and pathogenic proteobacteria and relation with strain biogeography. FEMS Microbiol. Ecol. 56: 455-470.

Bolhàr-Nordenkampf, H. R., and Öquist, G. 1993. Chlorophyll fluorescence as a tool in photosynthesis research. Pages 193-206 in: Photosynthesis and production in a changing environment: A field and laboratory manual. D. O. Hall, J. M. O. Scurlock, H. R. Bolhàr-Nordenkampf, R. C. Leegood, and S. P. Long, eds. Chapman and Hall, London.

Bonsall, R. F., Weller, D. M., and Thomashow, L. S. 1997. Quantification of 2,4-diacetylphloroglucinol produced by fluorescent Pseudomonas spp. in vitro and in the rhizosphere of wheat. Appl. Environ. Microbiol. 63: 951-955.

Brazelton, J. N., Pfeufer, E. E., Sweat, T. A., Gardener, B. B. M., and Coenen, C. 2008. 2,4-diacetylphloroglucinol alters plant root development. Mol. Plant-Microbe Interact 21:1349-1358.

Broekgaarden, C., Caarls, L., Vos, I. A., Pieterse, C. M., and Van Wees, S. C. 2015. Ethylene: Traffic controller on hormonal crossroads to defense. Plant Physiol. 169:2371-2379.

Bruto, M., Prigent-Combaret, C., Muller, D., and Moënne-Loccoz, Y. 2014 Analysis of genes contributing to plant-beneficial functions in Plant Growth-Promoting Rhizobacteria and related Proteobacteria. Sci. Rep. 4:6261.

Calderón, C. E., Pérez-García, A., de Vicente, A., and Cazorla, F. M. 2013. The dar genes of Pseudomonas chlororaphis PCL1606 are crucial for biocontrol activity via production of the antifungal compound 2-hexyl, 5-propyl resorcinol. Mol. Plant-Microbe Interact 26:554-565.

Cao, X. F., Linstead, P., Berger, F., Kieber, J., and Dolan, L. 1999 Differential ethylene sensitivity of epidermal cells is involved in the establishment of cell pattern in the Arabidopsis root. Physiol. Plant. 106: 311-317.

Choi, P. S., Naal, Z., Moore, C., Casado-Rivera, E., Abruña, H. D., Helmann, J. D., and Shapleigh, J. P. 2006. Assessing the impact of denitrifier-produced nitric oxide on other bacteria. Appl. Environ. Microbiol. 72:2200-2205.

Clifford, J. C., Buchanan, A., Vining, O., Kidarsa, T. A., Chang, J. H., McPhail, K. L., and Loper, J. E. 2016. Phloroglucinol functions as an intracellular and intercellular chemical messenger influencing gene expression in Pseudomonas protegens. Environ. Microbiol. 18: 3296-3308. 
Combes-Meynet, E., Pothier, J. F., Moënne-Loccoz, Y., and PrigentCombaret, C. 2011. The Pseudomonas secondary metabolite 2,4diacetylphloroglucinol is a signal inducing rhizoplane expression of Azospirillum genes involved in plant-growth promotion. Mol. PlantMicrobe Interact 24:271-284.

Couillerot, O., Combes-Meynet, E., Pothier, J. F., Bellvert, F., Challita, E., Poirier, M.-A., Rohr, R., Comte, G., Moënne-Loccoz, Y., and PrigentCombaret, C. 2011. The role of the antimicrobial compound 2,4diacetylphloroglucinol in the impact of biocontrol Pseudomonas fluorescens F113 on Azospirillum brasilense phytostimulators. Microbiology 157: 1694-1705.

Couillerot, O., Prigent-Combaret, C., Caballero-Mellado, J., and MoënneLoccoz, Y. 2009. Pseudomonas fluorescens and closely-related fluorescent pseudomonads as biocontrol agents of soil-borne phytopathogens. Lett. Appl. Microbiol. 48:505-512.

Couillerot, O., Ramírez-Trujillo, A., Walker, V., von Felten, A., Jansa, J., Maurhofer, M., Défago, G., Prigent-Combaret, C., Comte, G., CaballeroMellado, J., and Moënne-Loccoz, Y. 2013. Comparison of prominent Azospirillum strains in Azospirillum-Pseudomonas-Glomus consortia for promotion of maize growth. Appl. Microbiol. Biotechnol. 97:4639-4649.

Cristescu, S. M., Mandon, J., Arslanov, D., De Pessemier, J., Hermans, C. and Harren, F. J. M. 2013. Current methods for detecting ethylene in plants. Ann. Bot. (Lond.) 111:347-360.

Cronin, D., Moënne-Loccoz, Y., Fenton, A., Dunne, C., Dowling, D. N., and O'Gara, F. 1997a. Ecological interaction of a biocontrol Pseudomonas fluorescens strain producing 2,4-diacetylphloroglucinol with the soft rot potato pathogen Erwinia carotovora subsp. atroseptica. FEMS Microbiol. Ecol. 23:95-106.

Cronin, D., Moenne-Loccoz, Y., Fenton, A., Dunne, C., Dowling, D. N., and O'gara, F. 1997b. Role of 2,4-diacetylphloroglucinol in the interactions of the biocontrol pseudomonad strain F113 with the potato cyst nematode Globodera rostochiensis. Appl. Environ. Microbiol. 63: 1357-1361.

De La Fuente, L., Landa, B. B., and Weller, D. M. 2006. Host crop affects rhizosphere colonization and competitiveness of 2,4-diacetylphloroglucinolproducing Pseudomonas fluorescens. Phytopathology 96:751-762.

de Weert, S., Vermeiren, H., Mulders, I. H. M., Kuiper, I., Hendrickx, N., Bloemberg, G. V., Vanderleyden, J., De Mot, R., and Lugtenberg, B. J. 2002. Flagella-driven chemotaxis towards exudate components is an important trait for tomato root colonization by Pseudomonas fluorescens. Mol. Plant-Microbe Interact 15:1173-1180.

de Werra, P., Huser, A., Tabacchi, R., Keel, C., and Maurhofer, M. 2011. Plant- and microbe-derived compounds affect the expression of genes encoding antifungal compounds in a pseudomonad with biocontrol activity. Appl. Environ. Microbiol. 77:2807-2812.

de Werra, P., Péchy-Tarr, M., Keel, C., and Maurhofer, M. 2009. Role of gluconic acid production in the regulation of biocontrol traits of Pseudomonas fluorescens CHA0. Appl. Environ. Microbiol. 75:4162-4174.

Dunne, C., Moënne-Loccoz, Y., McCarthy, J., Higgins, P., Powell, J., Dowling, D. N., and O'Gara, F. 1998. Combining proteolytic and phloroglucinol-producing bacteria for improved biocontrol of Pythiummediated damping-off of sugar beet. Plant Pathol. 47:299-307.

Fernández-Marcos, M., Sanz, L., Lewis, D. R., Muday, G. K., and Lorenzo, O. 2011. Nitric oxide causes root apical meristem defects and growth inhibition while reducing PIN-FORMED 1 (PIN1)-dependent acropetal auxin transport. Proc. Natl. Acad. Sci. U.S.A. 108:18506-18511.

Flury, P., Aellen, N., Ruffner, B., Péchy-Tarr, M., Fataar, S., Metla, Z., Dominguez-Ferreras, A., Bloemberg, G., Frey, J., Goesmann, A., Raaijmakers, J. M., Duffy, B., Höfte, M., Blom, J., Smits, T. H., Keel, C., and Maurhofer, M. 2016. Insect pathogenicity in plant-beneficial pseudomonads: Phylogenetic distribution and comparative genomics. ISME J. 10:2527-2542.

Galland, M., Gamet, L., Varoquaux, F., Touraine, B., Touraine, B., and Desbrosses, G. 2012. The ethylene pathway contributes to root hair elongation induced by the beneficial bacteria Phyllobacterium brassicacearum STM196. Plant Sci. 190:74-81.

Ghiglione, J.-F., Richaume, A., Philippot, L., and Lensi, R. 2002. Relative involvement of nitrate and nitrite reduction in the competitiveness of Pseudomonas fluorescens in the rhizosphere of maize under non-limiting nitrate conditions. FEMS Microbiol. Ecol. 39:121-127.

Glick, B. R., Penrose, D. M., and Li, J. 1998. A model for the lowering of plant ethylene concentrations by plant growth-promoting bacteria. J. Theor. Biol. 190:63-68.

Haichar, F. Z., Heulin, T., Guyonnet, J. P., and Achouak, W. 2016. Stable isotope probing of carbon flow in the plant holobiont. Curr. Opin. Biotechnol. 41:9-13.

Hochholdinger, F., and Zimmermann, R. 2008. Conserved and diverse mechanisms in root development. Curr. Opin. Plant Biol. 11:70-74.
Højberg, O., Schnider, U., Winteler, H. V., Sørensen, J., and Haas, D. 1999. Oxygen-sensing reporter strain of Pseudomonas fluorescens for monitoring the distribution of low-oxygen habitats in soil. Appl. Environ. Microbiol. 65:4085-4093.

Honma, M., and Shimomura, T. 1978. Metabolism of 1-aminocyclopropane-1carboxylic acid. Agric. Biol. Chem. 42:1825-1831.

Iavicoli, A., Boutet, E., Buchala, A., and Métraux, J.-P. 2003. Induced systemic resistance in Arabidopsis thaliana in response to root inoculation with Pseudomonas fluorescens CHA0. Mol. Plant-Microbe Interact 16:851-858.

Jung, J. K. H., and McCouch, S. 2013. Getting to the roots of it: Genetic and hormonal control of root architecture. Front. Plant Sci. 4:186.

Kidarsa, T. A., Goebel, N. C., Zabriskie, T. M., and Loper, J. E. 2011. Phloroglucinol mediates cross-talk between the pyoluteorin and 2,4diacetylphloroglucinol biosynthetic pathways in Pseudomonas fluorescens Pf-5. Mol. Microbiol. 81:395-414.

Lanteigne, C., Gadkar, V. J., Wallon, T., Novinscak, A., and Filion, M. 2012. Production of DAPG and HCN by Pseudomonas sp. LBUM300 contributes to the biological control of bacterial canker of tomato. Phytopathology 102:967-973.

Lemanceau, P., Blouin, M., Muller, D., and Moënne-Loccoz, Y. 2017. Let the core microbiota be functional. Trends Plant Sci. 22:583-595.

Lombardo, M. C., Graziano, M., Polacco, J. C., and Lamattina, L. 2006. Nitric oxide functions as a positive regulator of root hair development. Plant Signal. Behav. 1:28-33.

Lombardo, M. C., and Lamattina, L. 2012. Nitric oxide is essential for vesicle formation and trafficking in Arabidopsis root hair growth. J. Exp. Bot. 63:4875-4885.

Loper, J. E., Hassan, K. A., Mavrodi, D. V., Davis, E. W., 2nd, Lim, C. K. Shaffer, B. T., Elbourne, L. D., Stockwell, V. O., Hartney, S. L., Breakwell, K., Henkels, M. D., Tetu, S. G., Rangel, L. I., Kidarsa, T. A. Wilson, N. L., van de Mortel, J. E., Song, C., Blumhagen, R., Radune, D., Hostetler, J. B., Brinkac, L. M., Durkin, A. S., Kluepfel, D. A., Wechter, W. P., Anderson, A. J., Kim, Y. C., Pierson, L. S., 3rd, Pierson, E. A., Lindow, S. E., Kobayashi, D. Y., Raaijmakers, J. M., Weller, D. M., Thomashow, L. S., Allen, A. E., and Paulsen, I. T. 2012. Comparative genomics of plant-associated Pseudomonas spp.: Insights into diversity and inheritance of traits involved in multitrophic interactions. PLoS Genet. 8:e1002784.

Lugtenberg, B. J. J., and Dekkers, L. C. 1999. What makes Pseudomonas bacteria rhizosphere competent? Environ. Microbiol. 1:9-13.

Mantelin, S., Desbrosses, G., Larcher, M., Tranbarger, T. J., Cleyet-Marel, J.-C., and Touraine, B. 2006. Nitrate-dependent control of root architecture and $\mathrm{N}$ nutrition are altered by a plant growth-promoting Phyllobacterium sp. Planta 223:591-603.

Marx, C. J., and Lidstrom, M. E. 2002. Broad-host-range cre-lox system for antibiotic marker recycling in gram-negative bacteria. Biotechniques 33: 1062-1067.

Miller, S. H., Browne, P., Prigent-Combaret, C., Combes-Meynet, E., Morrissey, J. P., and O'Gara, F. 2010. Biochemical and genomic comparison of inorganic phosphate solubilization in Pseudomonas species. Environ. Microbiol. Rep. 2:403-411.

Pitts, R. J., Cernac, A., and Estelle, M. 1998. Auxin and ethylene promote root hair elongation in Arabidopsis. Plant J. 16:553-560.

Pothier, J. F., Wisniewski-Dyé, F., Weiss-Gayet, M., Moënne-Loccoz, Y., and Prigent-Combaret, C. 2007. Promoter-trap identification of wheat seed extract-induced genes in the plant-growth-promoting rhizobacterium Azospirillum brasilense Sp245. Microbiology 153:3608-3622.

Prigent-Combaret, C., Blaha, D., Pothier, J. F., Vial, L., Poirier, M.-A., Wisniewski-Dyé, F., and Moënne-Loccoz, Y. 2008. Physical organization and phylogenetic analysis of $a c d R$ as leucine-responsive regulator of the 1-aminocyclopropane-1-carboxylate deaminase gene acdS in phytobeneficial Azospirillum lipoferum 4B and other proteobacteria. FEMS Microbiol. Ecol. 65:202-219.

Ramette, A., Frapolli, M., Fischer-Le Saux, M., Gruffaz, C., Meyer, J. M., Défago, G., Sutra, L., and Moënne-Loccoz, Y. 2011. Pseudomonas protegens sp. nov., widespread plant-protecting bacteria producing the biocontrol compounds 2,4-diacetylphloroglucinol and pyoluteorin. Syst. Appl. Microbiol. 34:180-188.

Ramette, A., Moënne-Loccoz, Y., and Défago, G. 2003. Prevalence of fluorescent pseudomonads producing antifungal phloroglucinols and/or hydrogen cyanide in soils naturally suppressive or conducive to tobacco black root rot. FEMS Microbiol. Ecol. 44:35-43.

Redondo-Nieto, M., Barret, M., Morrissey, J., Germaine, K., MartínezGranero, F., Barahona, E., Navazo, A., Sánchez-Contreras, M., Moynihan, J. A., Muriel, C., Dowling, D., O’Gara, F., Martín, M., and Rivilla, R. 2013. Genome sequence reveals that Pseudomonas fluorescens F113 possesses a large and diverse array of systems for rhizosphere function and host interaction. BMC Genomics 14:54. 
Richardson, A. E., Barea, J.-M., McNeill, A. M., and Prigent-Combaret, C. 2009. Acquisition of phosphorus and nitrogen in the rhizosphere and plant growth promotion by microorganisms. Plant Soil 321:305-339.

Romanowski, A., Migliori, M. L., Valverde, C., and Golombek, D. A. 2011 Circadian variation in Pseudomonas fluorescens (CHA0)-mediated paralysis of Caenorhabditis elegans. Microb. Pathog. 50:23-30.

RStudio Team. 2015. RStudio: Integrated Development for R. RStudio, Inc., Boston.

Sánchez-Contreras, M., Martín, M., Villacieros, M., O’Gara, F., Bonilla, I., and Rivilla, R. 2002. Phenotypic selection and phase variation occur during alfalfa root colonization by Pseudomonas fluorescens F113. J. Bacteriol. 184:1587-1596.

Schnider-Keel, U., Seematter, A., Maurhofer, M., Blumer, C., Duffy, B., Gigot-Bonnefoy, C., Reimmann, C., Notz, R., Défago, G., Haas, D., and Keel, C. 2000. Autoinduction of 2,4-diacetylphloroglucinol biosynthesis in the biocontrol agent Pseudomonas fluorescens $\mathrm{CHA} 0$ and repression by the bacterial metabolites salicylate and pyoluteorin. J. Bacteriol. 182:1215-1225.

Shanahan, P., O'sullivan, D. J., Simpson, P., Glennon, J. D., and O'gara, F. 1992. Isolation of 2,4-diacetylphloroglucinol from a fluorescent pseudomonad and investigation of physiological parameters influencing its production. Appl. Environ. Microbiol. 58:353-358.

Simon, A., and Ridge, E. H. 1974. The use of ampicillin in a simplified selective medium for the isolation of fluorescent pseudomonads. J. Appl. Bacteriol. 37:459-460.

Spaepen, S., and Vanderleyden, J. 2011. Auxin and plant-microbe interactions. Cold Spring Harb. Perspect. Biol. 3:a001438.

Stella, M., and Halimi, M. S. 2015. Gluconic acid production by bacteria to liberate phosphorus from insoluble phosphate complexes. J. Trop. Agric. Fd. Sc. 43:41-53.

Street, I. H., Aman, S., Zubo, Y., Ramzan, A., Wang, X., Shakeel, S. N., Kieber, J. J., and Schaller, G. E. 2015. Ethylene inhibits cell proliferation of the Arabidopsis root meristem. Plant Physiol. 169:338-350.

Swarup, R., Perry, P., Hagenbeek, D., Van Der Straeten, D., Beemster, G. T. S., Sandberg, G., Bhalerao, R., Ljung, K., and Bennett, M. J. 2007.
Ethylene upregulates auxin biosynthesis in Arabidopsis seedlings to enhance inhibition of root cell elongation. Plant Cell 19: 2186-2196.

Vacheron, J., Combes-Meynet, E., Walker, V., Gouesnard, B., Muller, D., Moënne-Loccoz, Y., and Prigent-Combaret, C. 2016a. Expression on roots and contribution to maize phytostimulation of 1-aminocyclopropane1-decarboxylate deaminase gene acdS in Pseudomonas fluorescens F113. Plant Soil 407:187-202.

Vacheron, J., Desbrosses, G., Bouffaud, M.-L., Touraine, B., MoënneLoccoz, Y., Muller, D., Legendre, L., Wisniewski-Dyé, F., and PrigentCombaret, C. 2013. Plant growth-promoting rhizobacteria and root system functioning. Front. Plant Sci. 4:356.

Vacheron, J., Moënne-Loccoz, Y., Dubost, A., Gonçalves-Martins, M., Muller, D., and Prigent-Combaret, C. 2016b. Fluorescent Pseudomonas strains with only few plant-beneficial properties are favored in the maize rhizosphere. Front. Plant Sci. 7:1212.

Vessey, J. K. 2003. Plant growth promoting rhizobacteria as biofertilizers. Plant Soil 255:571-586.

Villacieros, M., Power, B., Sánchez-Contreras, M., Lloret, J., Oruezabal, R. I., Martín, M. 2003. Colonization behaviour of Pseudomonas fluorescens and Sinorhizobium meliloti in the alfalfa (Medicago sativa) rhizosphere. Plant Soil 251:47-54.

Voisard, C., Keel, C., Haas, D., and Dèfago, G. 1989. Cyanide production by Pseudomonas fluorescens helps suppress black root rot of tobacco under gnotobiotic conditions. EMBO J. 8:351-358.

Von Felten, A., Défago, G., and Maurhofer, M. 2010. Quantification of Pseudomonas fluorescens strains F113, CHA0 and Pf153 in the rhizosphere of maize by strain-specific real-time PCR unaffected by the variability of DNA extraction efficiency. J. Microbiol. Methods 81: 108-115.

Walker, V., Couillerot, O., Felten, A. V., Bellvert, F., Jansa, J., Maurhofer, M. 2012. Variation of secondary metabolite levels in maize seedling roots induced by inoculation with Azospirillum, Pseudomonas and Glomus consortium under field conditions. Plant Soil 356:151-163. 